\title{
A Feature Computation Tree Model to Specify Requirements and Reuse
}

Citation for published version (APA):

Roubtsova, E. E., \& Roubtsov, S. A. (2006). A Feature Computation Tree Model to Specify Requirements and Reuse. In J. Filipe, Y. Manolopoulos, P. Constantopoulos, \& J. Cordeiro (Eds.), Proceedings of the Eighth International Conference on Enterprise Information Systems - (Volume 5) (Vol. 5, pp. 118-125). SCITEPRESSScience and Technology Publications, Lda.. https://doi.org/10.5220/0002443401180125

\section{DOI:}

$10.5220 / 0002443401180125$

Document status and date:

Published: 01/05/2006

Document Version:

Publisher's PDF, also known as Version of record

Please check the document version of this publication:

- A submitted manuscript is the version of the article upon submission and before peer-review. There can be important differences between the submitted version and the official published version of record. People interested in the research are advised to contact the author for the final version of the publication, or visit the DOI to the publisher's website.

- The final author version and the galley proof are versions of the publication after peer review.

- The final published version features the final layout of the paper including the volume, issue and page numbers.

Link to publication

\section{General rights}

Copyright and moral rights for the publications made accessible in the public portal are retained by the authors and/or other copyright owners and it is a condition of accessing publications that users recognise and abide by the legal requirements associated with these rights.

- Users may download and print one copy of any publication from the public portal for the purpose of private study or research.

- You may not further distribute the material or use it for any profit-making activity or commercial gain

- You may freely distribute the URL identifying the publication in the public portal.

If the publication is distributed under the terms of Article 25fa of the Dutch Copyright Act, indicated by the "Taverne" license above, please follow below link for the End User Agreement:

https://www.ou.nl/taverne-agreement

Take down policy

If you believe that this document breaches copyright please contact us at:

pure-support@ou.nl

providing details and we will investigate your claim.

Downloaded from https://research.ou.nl/ on date: 26 Apr. 2023 


\title{
A FEATURE COMPUTATION TREE MODEL TO SPECIFY REQUIREMENTS AND REUSE
}

\author{
E.E.Roubtsova \\ Open University of the Netherlands \\ Postbus 2960, 6401DL, Heerlen, the Netherlands \\ Ella.Roubtsova@ou.nl \\ S.A.Roubtsov \\ Technical University Eindhoven \\ Postbus 513, 5600MB, Eindhoven, the Netherlands \\ S.Roubtsov@tue.nl
}

Keywords: $\quad$ Requirements, feature computation tree, composition, formal methods, goals of reuse, methodology, specification, temporal logic

Abstract: A large subset of requirements for complex systems, services and product lines is traditionally specified by hierarchical structures of features. Features are usually gathered and represented in the form of a feature tree. The feature tree is a structural model. It represents mainly composition and specialization relations between features and does not provide the possibility to specify requirements in the form of ordering relations defined on functional features. Use case scenarios are usually employed for specification of the ordering relations. However, use case scenarios comprise isolated sequences of features, and therefore they may be inconsistent and even may contradict each other and the feature tree. Moreover, some use case scenarios defining relations on features may be incomplete.

In order to support consistent specification of requirements, we suggest using a pair of related models: a feature tree model and a feature computation tree model. The pair of such related feature tree models provides the basis for the method of consistency checks of requirements. It introduces a united view on the system's behavior at the stage of requirement specification and facilitates specification of forbidden sequences and construction complete sequences from incomplete ones. It allows designers to precisely specify the desired reuse and to find that a certain sort of reuse is not possible. Understanding already at the stage of requirements engineering that a subsystem cannot be reused without modification saves effort and money spent on development. The proposed method and models are explained using a case study of design of a system for electronic cards production.

\section{INTRODUCTION}

Building configurable and reusable systems is a promising way to tackle the problem of software evolution. Configuration and reuse are achievable after gathering system requirements and relating them in such a manner that would provide the possibility of avoiding design errors at an early stage of a system's life cycle.

The textual specification of requirements is the most common form for requirements engineering. However, it supports neither a proper overview of the requirements for the system under design (Felty and Namijoshi, 2003; Muller, 2004) nor a requirements reference model for configurable systems such as, for example, product lines. A possible alternative proposed in this area is a feature tree model (Griss et al., 1998; Svahnberg et al., 2002; van Gurp, 2003), which allows for a system's functional requirements to be gathered and presented in a compact and visualized form.

However, the feature tree model does not solve all the problems of requirements specification for configurable systems. First of all, this model is structural, it represents mainly composition and specialization relations between functional requirements-features and does not provide the possibility to specify requirements in the form of ordering relations defined on functional features. For this purpose use case scenarios and UML sequence diagrams are traditionally employed (Cockburn, 2000). However, use case scenarios or UML sequence diagrams (OMG, 2003; Douglass, 2003) consider isolated sequences of features. Each sequence presents only one path of the system's behavior. So, such sequences can be inconsistent and even contradict each other (Felty and Namijoshi, 2003) as well as the feature tree model. A united view on the system's behavior, similar to that which the 
feature tree model provides on the system's structure, usually does not exist at the stage of requirements specification.

The second problem is that any use case specification inevitably suffers from excessively 'positive' attitude of users and requirements engineers: they tend to specify what the system has to do and forget to specify what the system should not do. As a result, very often some undesired system behavior remains undefined at the requirements specification stage.

Thirdly, the goal of system reuse, although very well understood as desirable, often remains specified only informally because the feature tree model, being structural and static, has its limitations regarding behavioral aspects of reuse.

Thus, the feature tree model needs to be accompanied by some behavioral model which would help requirement engineers build a mental image of a system's behavior in terms of features and their reuse. The model should be related to the feature tree model, has to present the behavior of the system at a high level of abstraction, and needs to be rigorous enough to enable the use of formal methods to check for consistency of requirements and define the desired reuse.

In this paper, we propose using a pair of related models: a feature tree model and a feature computation tree model. We name the proposed behavioral model a feature computation tree model to emphasize its connection to the traditional feature tree model. The pair of such feature models solves all the three problems mentioned above.

1. It provides the basis for the method of consistency checks of requirements that we propose in this paper.

2. It introduces a united view on a system's behavior at the stage of requirement specification and facilitates the specification of forbidden behavior.

3 . It allows designers to precisely specify the desired reuse and to show that some sort of reuse is not possible. Understanding already at the stage of requirements engineering that a subsystem cannot be reused without modification saves effort and money spent on development.

We present tool support for all three tasks. The tool provides the building of a feature computation tree from sequences constructed from requirements and features of the traditional feature tree. The tool provides automated consistency checks of requirements. Revision and extension of the set of requirements is also supported. The tool also provides several patterns of reuse to help designers understand and precisely formulate the desired reuse.

The paper is aimed to demonstrate the proposed models and methods in use. The approach is explained using a case study of a modular system for production of electronic cards, and therefore some of the formal definitions are replaced by informal explanations. Section 2 describes the feature tree of a mod- ular system for production of electronic cards. The section also presents the requirements for the system. The approaches to consistency checks of requirements, to specification of forbidden sequences and to formalization of the notion of reuse are demonstrated in Section 3. Section 4 discusses tool support for the proposed models and methods. Section 5 presents related work and conclusions.

\section{A MODULAR SYSTEM FOR PRODUCTION OF ELECTRONIC CARDS}

To explain the approach proposed in this paper, we use an example of the modular system for production of electronic cards DC-9000 (DATACARD, 2005), as one of the authors was involved in resolving conflicts of requirements and configurations of such a system.

A system for production of electronic cards is composed from different modules to provide needs of different users. Some users of this system need to produce anonymous cards storing a certain amount of electronic money. Other users produce personalized cards providing access to an account number, and having a name, an address and sometimes a photo of a card holder on their surface.

A card is ready, if it is correctly initialized electronically and, in case of a personalized card, it is printed correctly (correct magnetic stripe, thermal printing of the text and graphics) and the information about this card is saved in a database for further tracing the card during its entire life cycle, e.g. for performing transactions with the card holder's account, blocking of stolen or lost cards, etc.

The DC-9000 equipment allows for any specific features required for a user to be introduced into the system not only by using different hardware modules but also through functions of its application interface. A variety of hardware signals is sent by different modules after card production completion or in the event of failure. A specific user application can utilize those signals in a different way, e.g., as triggers for sending data images of produced cards to a database or, otherwise, for sorting out defective cards.

Abstracting from all details of this complex process, we consider the following important functional features of the system:

- $A C$ - production of an anonymous e-card;

- $P C$ - personalization of an e-card. This implies writing into the card electronic personal data (subfeature $P C_{e}$ ) and printing personal data onto the card surface (sub-feature $P C_{p}$ );

- $D B$ - registration of an anonymous card in the database; 
- $D B^{p}$ - registration of a personal card in the database;

- $D F$ - separation of defective anonymous cards.

- $D F^{p}$ - separation of defective personal cards. Separation is required both when the card is incorrect electronically (sub-feature $D F_{e}$ ) and in case of printing failure (sub-feature $D F_{p}$ ).
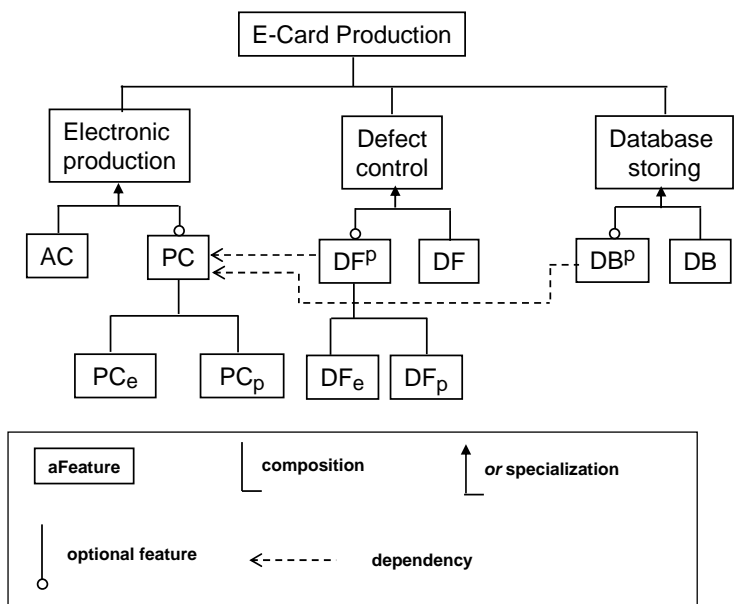

Figure 1: Traditional feature tree

Figure 1 presents the functional features of the system in the form of a traditional feature tree (Griss et al., 1998; Svahnberg et al., 2002; van Gurp, 2003). A traditional feature tree allows for representing compositions of features, specialization (inheritance) relations on features as well as mutual dependencies between features. In common case xor (not used in our example) and or specializations as well as optional features (both shown in Figure 1) allow designers to configure end-products differently providing variability of the system design.

The use of relations between features depends on the behavioral specification of an end-product. In particular, this means that the order in which features are implemented is also important. Obviously the correct order of high level features should be Electronic production, Defect control and Database storing (Figure 1). However, for the specialized features it is not so obvious. For example, features-inheritors $A C, D F, D B, P C_{p}, D F_{p}$, etc. in different configurations may have very different ordering relations, and this is not visible in the traditional feature tree.

Clarifying ordering relations of features at this abstract level of system presentation can prevent mistakes in specification and implementation. In practice such relations are established by use cases (Cockburn, 1997) specifying allowed sequences. Sometimes the sequences are incomplete. In the next section we demonstrate by example that such form of clarification of feature ordering leaves room for mistakes.

\subsection{Formalizing Requirements for the Systems Producing Anonymous and Personalized E-cards}

Requirements are usually specified textually (Muller, 2004). Requirements engineers can use the names of features from the feature tree and manipulate them as blocks to derive sequences from the text and present them as use cases (Cockburn, 2000), UML sequence diagrams (Cockburn, 1997) or sequential processes in the process algebra notation (Baeten and Weijland, 1990) or as property-formulas in the linear logic (Felty and Namijoshi, 2003; Berard et al., 2001).

Now, let us imagine that one of our customers needs to produce anonymous cards, and another one personalized cards.

The requirements of the customer who needs anonymous cards are the following:

1.1. A produced card is either registered in the database or separated as defective. The allowed sequences for the system producing anonymous cards are: $A C \cdot D B$ and $A C \cdot D F$. (The names of features are taken from Figure 1).

1.2. The information about defective cards should not appear in the database. The requirement is informal.

The requirements of the customer who needs to produce personalized cards are the following:

2.1. The system for production of personalized ecards is supposed to reuse the system for production of anonymous cards. The requirement is informal.

2.2. The personalized e-card is produced from an anonymous one by writing into the card additional electronic personal data (sub-feature $P C_{e}$ ). After that the personal data is printed onto the card surface (subfeature $P C_{p}$ ). So the incomplete allowed order is: $A C \cdot \ldots \cdot P C_{e} \cdot \ldots \cdot P C_{p}$.

2.3. Information about correctly produced personalized e-cards should appear in the database. The requirement is informal and can be understood differently. One understanding is that sequence $A C \cdot P C_{e}$. $D B^{p} \cdot P C_{p}$ is allowed. Another understanding is that sequence $A C \cdot P C_{e} \cdot P C_{p} \cdot D B^{p}$ should be implemented.

2.4. Defective cards are separated during production, so the allowed sequences are $A C \cdot D F ; A C \cdot P C_{e}$. $D F_{e}$; and $A C \cdot P C_{e} \cdot P C_{p} \cdot D F_{p}$.

In practice the requirements for both systems can be presented by use cases. Each use case is unfolded into a use case scenario (Cockburn, 2000). An example of a use case scenario for production of an anonymous card is shown in Figure 2. 


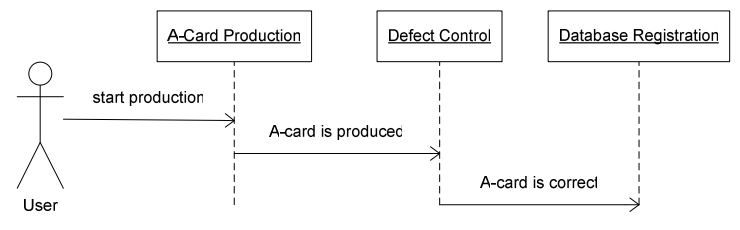

Figure 2: Use case scenario

The descriptions of requirements given above are the descriptions of the system features-requirements at the highest level. Presentation of the requirements as sequences is a necessary step in communication with customers. However, the set of sequences, presented, e.g., by use case scenarios, does not give a complete view on the system behavior because the sequences can be inconsistent in the sense that required sequences joined together can still produce forbidden behavior or even contain features unspecified in the feature tree of the system. So, the requirements should be checked on consistency.

Moreover, some requirements, for example, 1.2 (about forbidden sequences) and 2.1 (about reuse) are informal. What should be reused from the system producing anonymous cards? We can think of reusing separate features, sequences of features or the complete behavior of the first system. Another important question is: what may be reused without violation of the other requirements? Next section presents an approach to consistency checking of requirements and to formalizing the notion of reuse.

\section{AN APPROACH TO CONSISTENCY CHECKING OF REQUIREMENTS AND FORMALIZING THE NOTION OF REUSE}

Although the requirements are often incomplete, any attempt to build from them a picture of complete system behavior can be considered as a basis for consistency checking, for completing some incomplete requirements, for formalizing informal requirements and for retrieving new ones.

We suggest building a feature computation tree from allowed sequences which represents dynamics of the system in an abstract way. The feature computation tree built from features collected by the traditional feature tree can be further used to validate consistency of requirements and absence of forbidden features.

If an inconsistency is found during analysis of a feature computation tree, then the requirements are revised or new possible sequences are added to the tree. Some forbidden sequences can be generated from the feature computation tree to let a designer to make a decision if those sequences are really forbidden or missing in the set of requirements. As we will show later, the feature computation tree model also clarifies the requirement for desired reuse.

\subsection{Feature Computation Tree}

A feature computation tree is an abstract variant of a process tree (Baeten and Weijland, 1990), in which actions are replaces by functional features.

A feature computation tree $G_{p}=(A, N, E)$ is a graph which has a unique path from the node root to every other node. The root has no input edges.

- $A$ is a set of functional features.

The set of features of a system producing anonymous e-cards is $A=A C, D F, D B$.

- $N$ is a set of nodes, represented by points.

A node $n \in N$ of the tree represents an abstract state of a system under design. A node marks a starting and/or finishing points of a process implementing a feature. The root represents the initial state.

- $E$ is a set of arcs. An $\operatorname{arc} e=\left(n^{\prime}, n ", a\right) \in E$ of the tree is labelled by a feature name $a \in A$. An arc labelled by $a$ stands for a complete process implementing feature $a$. Process $a$ can be of different level of complexity, however, it always has a starting point represented on the feature computation tree by node $n^{\prime}$. The process can have several outputs, however, all of them can have an arc connecting each of them to an abstract final state of the process. Such a final state is represented on the feature computation tree by node $n "$. The final nodes has no output edges.

For example, in Figure 3, arc $A C$ stands for the process "Production of an anonymous e-card", $D F$ for the process "Separation of defective cards", and $D B$ represents the process "Registration of an anonymous card in the database".

Thus, a path in a feature computation tree is a sequence of arcs $\left(\left(n_{1}, n_{2}, a_{1}\right),\left(n_{2}, n_{3}, a_{2}\right), \ldots,\left(n_{m-1}, n_{m}, a_{m-1}\right)\right)$.

There is a unique sequence of features that corresponds to each path: $a_{1}, a_{2}, \ldots, a_{m-1}$. A path which starts from the root is called a root path.

If the designed behavior is cyclic, then a cycle is represented by two paths: one path for the cycle's body and the other path for the cycle's exit. Repeated cycle's bodies are replaced by dots: "...". An infinite sequence of features can be represented by its repetition rule, e.g. "abaabb...", allowing control over the progress of the repetitions. 


\subsection{Feature Computation Trees for the Systems Producing Anonymous and Personalized E-cards}

The high-level feature computation tree of the system for production of anonymous e-cards is shown in Figure 3. This tree combines two specified sequences 1.1. $A C \cdot D F$ and 1.2. $A C \cdot D B$ in such a way that, if the features in sequences are the same from the root, then they are merged. The algorithms of such merging is described in (Roubtsova and Kuiper, 2002).

As we can recognize in Figure 3, an anonymous e-card is successfully produced if its informational image is saved in the database of the system (process $D B$ is finished). Otherwise, if a defective ecard is produced, then the card is separated and the information about this card should not be kept in the database (process $D F$ is finished). So, from this tree we can derive two forbidden sequences $D F \cdot D B$ and $D B \cdot D F$ and formalize the requirement 1.2 , which says that the information about defective card should not be saved in the database. We deliberately oversimplified the picture, but even based on this picture, we have refined the initial requirements.

Building a feature computation tree from the sequences defined in the requirement 2.4 for the system producing personalized cards we get the feature computation tree shown in Figure 4a on the left hand side.

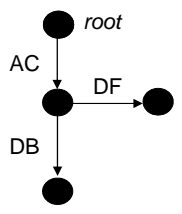

Figure 3: The feature computational tree for a system producing anonymous e-cards.

In order to prove that a sequence is consistent with a feature computation tree, we can use the method formally presented in (Roubtsova and Roubtsov, 2003). The method derives a computation tree from another computation tree using hiding and blocking techniques defined in process algebra (Baeten and Weijland, 1990). To prove that a sequence is consistent with a computation tree, it is required to find a path implementing this sequence on the tree. During derivation of the specific sequence the features starting other paths are blocked. Any sequence started by the blocked action is cut down. The features that do not belong to the specific sequence, but situated on the chosen path, are made invisible (hidden). Hidden features allow continuing the sequence of the chosen

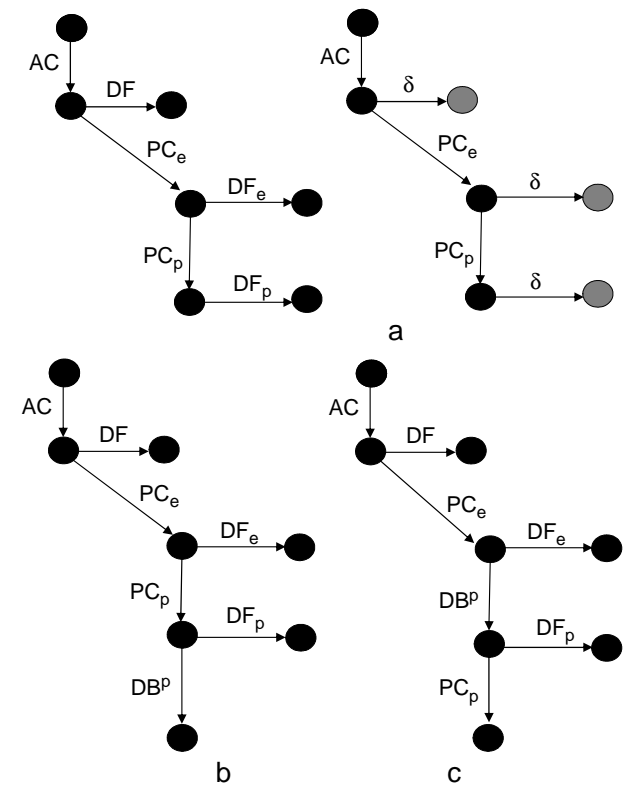

Figure 4: Feature computation trees for a system producing personalized e-cards.

path.

For example, in order to prove that the production sequence $A C \cdot \ldots \cdot P C_{e} \cdot \ldots \cdot P C_{p}$ (requirement 2.2) for the feature computation tree is presented in Figure $4 \mathrm{a}$, we block features $D F, D F_{e}, D F_{p}$ and have as the result the sequence $A C \cdot P C_{e} \cdot P C_{p}$ that matches with the production sequence (Figure $4 \mathrm{a}$, right hand side).

Two versions of the feature computation tree shown in Figures $4 \mathrm{~b}$ and $4 \mathrm{c}$ comply with the requirement 2.3 (regarding registration of correct cards in the database). This is a signal to a requirement engineer to choose one of them.

In order to make this choice, the forbidden sequences should be discovered by means of building all possible sequences of features from the feature computation tree and the textual requirements. For example, sequences $A C \cdot D B$ and $A C \cdot P C_{e} \cdot D B^{p}$ are not allowed because they save the information about incompletely produced and not printed cards. Sequences $A C \cdot D F \cdot D B, A C \cdot P C_{e} \cdot P C_{p} \cdot D F_{p} \cdot D B^{p}$ are not allowed because they save the information about defective cards.

The forbidden sequence $A C \cdot P C_{e} \cdot D B^{p}$ is implemented by the computation tree $4 \mathrm{c}$. It can be proven using the hiding and blocking technique. So, the feature computation tree (Figure 4b) with sequence $A C \cdot P C_{e} \cdot P C_{p} \cdot D B^{p}$ is accepted as the correct one for the system producing personalized cards. 


\subsection{Formalizing the Requirement for Reuse}

Now, the requirement 2.1 about reuse needs to be formalized.

The feature computation tree allows building some patterns of reuse.

The patterns of reuse manipulating feature computation trees are shown in Figure 5. Pattern (1) means extending all paths of a reused tree by a new tree. Pattern (2) presents extending one path of a reused tree by a new tree. Pattern (3) means inserting a new tree into all paths of a reused one. Pattern (4) implies inserting a new tree after a specific feature that appears in a reused tree.
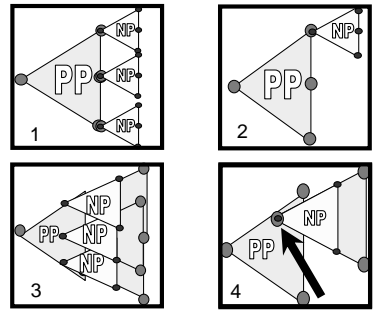

Figure 5: Reuse patterns. Feature computation trees are visualized as triangles with labels $P P$ and $N P$. $P P$ stands for a reused process and $N P$ - for a new process.

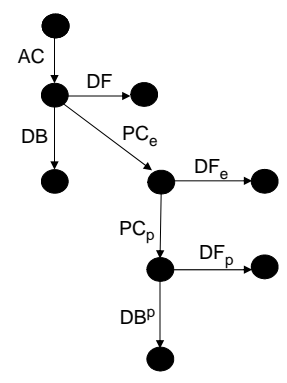

Figure 6: Feature computation tree for a system producing both types of e-cards. Case of incorrect reuse.

Building a system producing personalized cards we can insert a new feature computation tree after the feature $A C$ of the computation tree of the system producing anonymous cards (Figure 6). The resultant tree of such a design decision will include the forbidden sequence $A C \cdot D B$ identified in the previous subsection 3.2. So, we conclude that such complete reuse is incorrect. The only one path $A C * D F$ of the system producing anonymous cards can be reused. In order to produce a personalized card after the feature $A C$ only the feature $P C_{e}$ should be started.
Since the system for production of anonymous cards cannot distinguish the type of the card, a new feature computation tree that gives the type of the card should be built at the beginning of the process and one path of this tree should be extended by the feature computation tree shown in Figure 3 and the other - by the feature computation tree shown in Figure $4 \mathrm{~b}$. In other words, different feature computation trees and therefore systems should be built for production of cards of different types and for production of cards of both types.

\subsection{A Feature Computation Tree Model As an Internal Model for Other Models}

The features can be ordered using other popular specification notations like sequence diagrams (OMG, 2003), state charts (Harel and Kupferman, 2002) or Petri Nets (Murata, 1989; Kindler and Vesper, 1998). The two latter notations can combine sequences of features together. An example of the Petri Net model is shown in Figure 7. Building the Petri Net of the system we have assumed that transitions (boxes) are labelled by the names of features and places (cycles) represent the beginning and end states of processes implementing features.

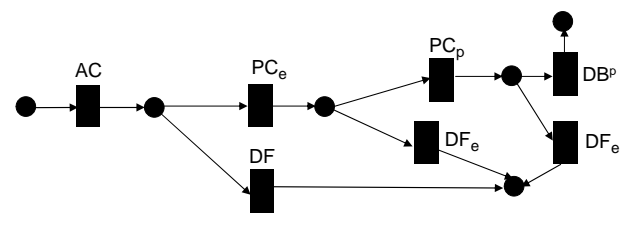

Figure 7: Petri Net for the system producing personalized e-cards

When it comes to consistency checks using formal model checking algorithms, all the models like state charts and Petri Nets are transformed into an internal computation tree model called in Petri Nets theory (Reisig, 1985) a reachability graph. At the feature-based level of abstraction this model is essentially a feature computation tree. So, introducing this model at the very beginning, we are able to use it as a basis for logic of reasoning, automated consistency checks and constructive notion of reuse.

That is why we suggest using the feature computation tree model for the requirement engineering. Besides, the feature computation model could be derived from any model used by the designer, it allows $\mathrm{him} / \mathrm{her}$ to formalize and validate consistency of requirements at a high level of abstraction. 


\section{TOOL SUPPORT}

In the area of requirements engineering there are three points of particular attention that are not covered by modern tools.

Firstly, the process of formalizing requirements is not covered by modern specification tools (Geppert and Schmid, 2002). Although there are tools that support proving formally written logical expressions (theorem provers) (Clarke et al., 1999; Berard et al., 2001), the step of getting logical expressions from informal requirements is not yet implemented.

Secondly, the precise design notations are weakly linked to the variety of requirements specification techniques, which makes it difficult to trace requirements within designs.

Thirdly, so far there was no support for specification of reuse goals.

We have made a tool-prototype that addresses all tree above mentioned issues.

1. The tool helps to formalize requirements as functional features and to specify desired or/and forbidden output related to the specified features.

The tool provides a list of available functional features derived from the system's feature tree. This list can be used by the requirements engineer to represent requirements as sequences.

The tool is able to build feature computation tree from given sequences and to check their consistency. Using the feature computation tree the set of statements about impossible outputs of the system is formulated and presented to the requirements engineer for control.

2. Designers of a system may traditionally use different standard notations. These notations should be related to the requirements. It has to be possible to trace them back to the requirements. As a large variety of specification notations can be transformed to our computation tree model, there is always a possibility to make such a transformation with tool support and to validate the results by the requirements. At the moment we have implemented support for transformation of a set of sequence diagrams to a feature computation tree.

3. We have proposed a taxonomy of reuse goals on the basis of a logic of reuse (Roubtsova and Roubtsov, 2004). The logic of reuse is aimed to express relations between the part of the system which is reused and the new part. The reused part is either a sequence or a subtree. The joining points are the nodes of the tree. Each node represents the end point of a successfully fulfilled feature functionality. The reuse relations are numerous but the types of computation tree logic formulas representing them are classified. In the tool, we use visual images of reuse patterns, like the ones shown in Figure 5. Choosing an image designer automatically gets the formal expression of the reuse goal and the corresponding procedure for correctness checks.

\section{RELATED WORK AND CONCLUSION}

To reduce the cost for and time spent on implementation, the possible conflicts of requirements should be found at the stage of requirements engineering.

The group of feature interaction detection approaches (Felty and Namijoshi, 2003; Cheng and Ohta, 1995) presents features by linear temporal logic formulas and checks their possible conflicts pair by pair. If a new requirement is added to the set of $n$ requirements then $n$ pairwise checks of requirements should be fulfilled. The feature interaction detection approaches do not create a general view on the designed system and do not formalize the notion of reuse suitable for the case.

The group of process-oriented approaches (Basten and van der Aalst, 2001; Wehrheim, 2002) do not relate modelling to requirements and do not formulate the desired reuse. This makes it difficult to use process-oriented approaches at the stage of requirements engineering and to validate consistency of requirements. This group of approaches uses an existential definition of reuse. It means that the process models of an old and a new system are produced and the old one should be derivable from the new one by hiding and blocking new actions in the new process model. This definition of reuse claims that if some sequence of hiding and blocking operations, such that allows deriving the old system can be found, then the new system reuses the old one. The existential approach can cause mistakes, because, in general, different sequences of hiding and blocking operations may allow deriving the old system, and the proven reuse may not correspond to the desired reuse. The process-oriented approaches do not answer the question as to how to apply hiding and blocking operations in order to prove the desired reuse.

We have defined a feature computation tree model to complement the traditional feature tree model. This makes it easier to formulate requirements in terms of sequences of features and to automate building a feature computation tree from such requirements. This prevents appearance of features that are unspecified in the feature tree. Due to the lack of room we have not formally defined the relation between the feature tree and the feature computation tree in the paper: it is a subject of another work.

The feature computation tree model supports the 
level of abstraction that is sufficient for requirements engineering. It generates an image of the system behavior, supports consistency checks of requirements and stimulates specification of forbidden sequences and other constraints. One class of such constraints is the class of reuse constraints. The combination of the feature computation tree model and the logic of reuse provides flexibility in the specification of reuse. Different reuse possibilities become clear and can be formalized with the help of the feature computation tree model. Moreover, with the help of the tree, the impossible reuse can be found.

We have applied our method so far for resolving conflicts of requirements and configurations at the implementation and testing stages of complex systems development. By means of this paper we would like to show how beneficial it would be to apply the approach at the stage of configuration specification or specification of system extensions.

\section{ACKNOWLEDGMENT}

The authors thank Prof. Jan Friso Groote from the Technical University Eindhoven, Prof. Mehmet Aksit, Dr. Arend Rensink and TRESE research group from the University of Twente and also Dr. Gerrit Muller from the Embedded Systems Institute (Eindhoven) for useful discussions of the paper.

\section{REFERENCES}

Baeten, J. and Weijland, W. (1990). Process Algebra. Cambridge University Press.

Basten, T. and van der Aalst, W. (2001). Inheritance of behaviour. The Journal of Logic and Algebraic Programming, 46:47-145.

Berard, B., Bidoit, M., Finkel, A., F.Laroussinie, Petit, A., Petrussi, L., Schnoebelen, P., and McKezie, P. (2001). Systems and Software Verification. Model-Checking Techniques and Tools. Springer-Verlag.

Cheng, K. and Ohta, T., editors (1995). Feature Interactions in Telecommunications III, October 11-13, 1995, Kyoto, Japan. IOS Press.

Clarke, E., Grumberg, O., and Peled, D. (1999). Model Checking. MIT Press, Cambridge, MA.

Cockburn, A. (1997). Structuring Use Cases with Goals. Journal of Object-Oriented Programming Sep-Oct and Nov-Dec.

Cockburn, A. (2000). Writing Effective Use Cases. Addison-Wesley.

DATACARD (2005). DATACARD 9000 Series System. Retrieved October 20, 2005 from http://www.identisys.com/documents.
Douglass, B. (2003). UML 2.0: Incremental Improvements for Scalability and Architecture. www.rtcmagazine.com/pdfs/2003/04/.

Felty, A. and Namijoshi, K. (2003). Feature Specification and Automated Conflict Detection. ACM Transactions on Software Engineering and Mothodology, 12(1):327 .

Geppert, B. and Schmid, K. (2002). Requirements Engineering for Product Lines. -An Overview- . In International Workshop on Requirements Engineering for Product Lines, REPL'02, pages 1-4, Essen, Germany.

Griss, M., Favaro, J., and d'Alessandro, M. (1998). Integrating feature modeling with the RSEB. In Fifth International Conference on Software Reuse (Cat. No.98TB100203), pages 76-85, Los Alamitos, CA, USA. IEEE Comput. Soc.

Harel, D. and Kupferman, O. (2002). On Object Systems and Behavioural Inheritance. IEEE Transactions On Software Engireering, 28(9):889-903.

Kindler, E. and Vesper, T. (1998). ESTL:A Temporal Logic for Events and States. In: Desel, J.; Silva LNCS 1420, ICATPN'98, pages 365-384.

Muller, G. (2004). CAFCR:AMulti-view Method for Embedded Systems Architecting:Balancing Genericity and Specificity. Technical University Enidhoven.

Murata, T. (1989). Petri Nets: Properties, Analysis and Applications . Proceedings of the IEEE, 77(4):541-580.

OMG (2003). UML 2.0 specification. Retrieved October 20, 2005 from http://www.omg.org/uml/.

Reisig, W. (1985). Petri Nets. An Introduction. SpringerVerlag.

Roubtsova, E. and Kuiper, R. (2002). Process semantics for UML component specifications to assess inheritance. ENTCS V 72(3), Editors P.Bottoni, M. Minas.

Roubtsova, E. and Roubtsov, S. (2003). UML-based Tool for Constructing Component Systems via Component Behaviour Inheritance. ENTCS V.80, Editors T.Erts, W. Fokkink.

Roubtsova, E. and Roubtsov, S. (2004). Constraints of Behavioural Inheritance. Springer LNCS 3047, Editors: Flavio Oquendo, Brian Warboys, pages 115-134.

Svahnberg, M., van Gurp, J., and Bosch, J. ( 2002). A Taxonomy of Variability Realization Techniques. Technical paper ISSN: 1103-1581, Blekinge Institute of Technology, Sweden.

van Gurp, J. (2003). On the Design and Presentation of Software Systems. Rijksuniversiteit Groningen, Groningen.

Wehrheim, H. (2002). Checking behavioural subtypes via refinement. In B. Jacobs and A. Rensink, editors, FMOODS 2002: Formal Methods for Open ObjectBased Distributed Systems, pages 79-93. 